\title{
The Dilemma of Raised Blood Levels of Folate and B12 in Autism
}

\section{Lonsdale*}

\section{Associate Emeritus, Cleveland Clinic Foundation, Cleveland, Ohio, USA}

\begin{abstract}
It has recently been reported that a raised level of folate and/or vitamin B12 in the blood of a newly parturient woman forecasts a marked increase in the possibility that the newborn infant will later develop autisma. The text that follows refers to the cases of two boys, each of which had suffered repeated episodes of acute febrile cervical lymphadenopathy. The medical history of one of the boys revealed that he had been previously admitted to a hospital during an episode of febrile lymphadenopathy. An enlarged cervical node had been removed for biopsy and it had been reported that its architecture, apart from its enlargement, was normal. In addition, it had been found that blood levels of folate and vitamin B12 were markedly increased. His mother had been accused of giving him too many supplements of vitamins, including folate and vitamin B12. She stated that she had not been giving the boy any vitamin supplements at all, a fact that was met with disbelief. Both boys had been indulged with sweets and each were found to have laboratory evidence of thiamin deficiency or abnormal status. One of the boys was studied in detail and the elevation of folate and B12 in the blood was confirmed. He was treated with large doses of thiamin as an outpatient. On his return the blood folate and vitamin B12 were found to be in the normal range. Thiamin therapy was discontinued and three weeks later he had another episode of febrile lymphadenopathy which was preceded by nocturnal sleepwalking accompanied by spontaneous enuresis. Blood folate and B12 were again elevated and thiamin treatment was restarted. With overall improvement in health and no further episodes of febrile lymphadenopathy, the folate and B12 levels were again normal when he returned for follow-up.
\end{abstract}

\section{Introduction}

In 2016, at the International Meeting for Autism Research in Baltimore it was reported that if a new mother had a very high level of blood folate right after giving birth, the risk that her child would develop autistic spectrum disorder (ASD) doubled. Very high levels of vitamin B12 tripled the risk. If both the levels of folate and B12 were high, the risk that the child would develop ASD increased by 17.6 times more than a mother with normal levels of both nutrients [1].

The cases of two boys with recurrent episodes of febrile cervical lymphadenopathy were published [2]. The medical history of one of them revealed that he had been admitted to a hospital for investigation during one of the episodes. Biopsy of an enlarged cervical node had yielded a report that, apart from its enlargement, it had normal architecture. In addition, he had been found to have increased concentrations of folate and vitamin B12 in the blood. The mother was accused of giving him too much supplementary folate and B12, although she stated that she had not been giving the boy any vitamin supplements.

Both boys had been indulged with sweets and evidence of thiamin deficiency or abnormal thiamin metabolism had been found, suggesting a therapeutic clinical trial with thiamin [2]. After large doses of thiamin had been given to both boys, the episodes of febrile lymphadenopathy ceased. The boy who had previously been found to have elevated blood levels of folate and B12 was studied in detail and these abnormal levels were con-

${ }^{*}$ Corresponding author: Derrick Lonsdale, MD, FAAP, FACN, Associate Emeritus, Cleveland Clinic Foundation, 28575 Westlake Village Dr. A 106, Westlake Ohio, Cleveland, Ohio, USA, Tel: 440-471-4852, E-mail: derricklonsdale@hotmail.com

Received: June 13, 2017; Accepted: June 18, 2018; Published online: June 20, 2018

Citation: Lonsdale D (2018) The Dilemma of Raised Blood Levels of Folate and B12 in Autism. J Neurodegener Disord 2(1):35-38

Copyright: @ 2018 Lonsdale D. This is an open-access article distributed under the terms of the Creative Commons Attribution License, which permits unrestricted use, distribution, and reproduction in any medium, provided the original author and source are credited. 
firmed before thiamin therapy. They both decreased into the normal range after thiamin treatment. Thiamin treatment was discontinued and three weeks later, an episode of sleepwalking with spontaneous enuresis was followed by another episode of febrile lymphadenopathy. Blood levels of folate and vitamin B12 were again raised and thiamin was restarted. Within a few days the blood levels of the two vitamins were back in the normal range and the large nymph nodes in the neck disappeared [2].

\section{Abnormal Thiamin Metabolism}

Thiamin deficiency has long been known to cause beriberi and the text that follows hypothesizes an explanation for why two boys expressed clinical thiamin deficiency as repeated episodes of febrile lymphadenopathy and its association with increased concentrations of folate and B12 in the blood [2]. One of the two boys had an abnormal erythrocyte transketolase thiamin pyrophosphate activity. The other boy who was studied in detail, had a substance in urine which had been reported to be related to thiamin triphosphate metabolism [3].

\section{Increased Concentrations of Serum Folate and B12}

Thiamine deficiency has a major effect on ATP synthesis [4]. Folate and B12 are both required in the energy consuming transmethylation pathway. It is hypothesized that they increase in the blood because they are both in their inactive form, or that there is a defect in the transmethylation pathway from energy insufficiency. All the biologic functions of folic acid are performed by tetrahydrofolate and other derivatives. Their biological availability depends upon dihydrofolate reductase (DHFR) action in liver. This action is unusually slow in humans, being less than $2 \%$ of that in rats, leading to the accumulation of unmetabolized folic acid [5]. It has been suggested that this low activity limits the conversion of fo-

${ }^{a}$ Daniele F Fallin and her group reported in 2013 that if a new mother has a very high level of folate right after giving birthmore than four times what is considered adequate-the risk that her child will develop a condition on the autism spectrum doubles. Very high vitamin B12 levels in new moms are also potentially harmful, tripling the risk that her offspring will develop an autism spectrum disorder. If both levels are extremely high, the risk that a child develops the condition increases 17.6 times.

Wendy Klag Center for Autism and Developmental Disabilities: The researchers found that 1 in 10 of the women had what is considered an excess amount of folate, while $6 \%$ had an excess amount of vitamin B12. Mothers who had very high blood levels of folate at delivery were twice as likely to have a child with autism compared to mothers with normal folate levels. Mothers with excessive B12 levels were 3 times as likely to have a child with autism. The risk was greatest among mothers who had excess levels of both folate and B12-their risk was over 17 times that of a mother with normal levels of both nutrients. late acid into its biologically active form when folic acid is consumed in excess as a supplement. However, DHFR activity also requires reducing equivalents, supplied by the hexose monophosphate shunt, which is dependent upon thiamine-dependent transketolase [6]. Autism has been called a redox/methylation disorder [7]. Treatments that could address redox metabolism abnormalities include methylcobalamin, with and without folinic acid. Both open-label double-blind, placebo-controlled studies have reported improvement in ASD symptoms with tetrahydrobiopterin [8]. Serum vitamin B12 levels were determined in a group of 51 parturients, in their newborn infants and the placentas. The infants had 2.3 times higher vitamin B12 levels than the mothers and the concentration of this vitamin in the intervillous space of the placenta was greater than that in the blood of both newborns and mothers. The relationship between folate and vitamin B12 concentrations in the maternal, fetal and placental sera was investigated. Higher serum folate concentrations were reported in the group of parturients who had received vitamin B12 supplementation during pregnancy, indicating the close metabolic interrelationship between B12 and folate [9].

\section{The Potential Relationship with Thiamin}

Kawasaki disease has been reported to occur with cervical lymphadenopathy as the sole initial presentation [10]. A possible diagnosis of Kawasaki disease, progressing to biotin-responsive basal ganglia disease, arising from a mutation in the SLC 19 A3 gene encoding the human thiamine transporter 2 protein has also been reported [11]. Neurologic and hematologic abnormalities are common in HIV-infected children. Folate and B12 levels were abstracted from the medical records of $103 \mathrm{HIV}$ infected children. All of them had serum folate or B12 levels within or above the normal range. Children with elevated B12 were significantly more likely to be younger and have higher mean folate levels compared with children who had a normal serum B12. None of the patients had been taking multivitamins or manifested clinical evidence of gastrointestinal malabsorption [12]. Thiamine deficiency was found in nine of 39 patients with AIDS or AIDS-related complex [13].

Thiamin and related metabolite status was studied in the plasma and urine of autistic children and compared with healthy controls. Plasma thiamin and thiamin monophosphate concentrations were similar in both study groups, but thiamin pyrophosphate was decreased by $24 \%$ in autistic children. Plasma protein dityrosine content was increased $88 \%$ while other oxidative markers were unchanged [14]. Dityrosine (3,3-bityrosine) was identified as the cross-link in oxidized proteins [15]. Autistic spectrum disorder (ASD) is marked by a complex interaction between environmental factors and genetic 
predisposition. Evidence for thiamin homeostasis, heavy metal deposition and cellular immunity all have a role [16].

\section{The Potential Role of Malnutrition in Autism}

Autism rates in the United States are reported to be increasing at a rate of $15 \%$ per year. This surely indicates common environmental factors, including malnutrition. A study of the Women, Infants, and Children (WIC) program was published. Autism rates for each state were established and the percentage of infants participating in the WIC program for low-income families was calculated for each of the 50 states. The states with the highest WIC participation had significantly lower autism rates. Infants who were solely breasts-fed had diets that contained less thiamin, riboflavin and vitamin $\mathrm{D}$ than the minimal daily requirements, implicating maternal malnutrition [17].

An 11-year-old autistic boy was hospitalized with hepatomegaly and liver dysfunction as well as lactic acidosis. His diet had been severely limited and was deficient in multiple micronutrients, including thiamin and pyridoxine. Lactic acid decreased rapidly with administration of thiamin and status epilepticus was associated with low serum pyridoxine. This was also resolved by the administration of pyridoxine [18]. Brain damage in infantile autism may involve the same complex of brainstem nuclei that are damaged by alcohol abuse, thiamin deficiency, and asphyxia. They are metabolically the most active structures in the brain, making them vulnerable to many injurious factors. If this control is lost it may result in the defects of awareness and responsiveness seen in autistic children [19]. Of parents of ASD children, 79\% reported some concern regarding their feeding behavior compared to $64 \%$ of parents of typically developing children. A significantly greater proportion of ASD children failed to meet the estimated average requirement of thiamin, vitamin C, and copper [20]. An uncontrolled pilot study reported clinical benefit in 8 of 10 autistic children with thiamin tetrahydrofurfuryl disulfide (TTFD) [21]. Due to genetic defects or illness some individuals require higher amounts of thiamin than are typically provided by the diet and TTFD treatment resulted in increased whole brain thiamin concentration in juvenile male DBA/2J mice [22].

\section{Conclusion}

Thiamin deficiency, caused by poor diet or by genetic abnormality, causes its effects by damaging energy metabolism. That is why beriberi has so many symptoms, none of which are pathognomonic. Its major effects are diverse and the brain and heart are major targets because of their high rate of metabolism. Furthermore, the hypo- thalamic, autonomic, endocrine axis is peculiarly sensitive to thiamin deficiency. Thus, the expression of disease is variable and depends on the degree of deficiency and how long it has been present. It is the biochemical lesion that matters [23]. For example, it is becoming clear that diabetes is highly influenced by high-dose thiamin and its derivatives [24,25]. Thus, a number of diseases, if not directly caused by, are influenced by thiamin deficiency that is likely to be missed without a high level of clinical insight.

We hypothesize that energy deficiency in these two boys invoked a strategic defense process in the form of febrile lymphadenopathy, initiated by the brain under the false impression of an attack by a microorganism. The energy deficiency affected the normal function of transmethylation, causing folate and vitamin B12 to accumulate. With restoration of adequate energy metabolism by the use of thiamin, the normal activity of transmethylation resumed.

\section{References}

1. Desmon S (2016) Too much folate in pregnant women increases autism risk, study suggests.

2. Lonsdale D (1980) Recurrent febrile lymphadenopathy treated with large doses of vitamin B1: Report of two cases. Dev Pharmacol Ther 1: 254-264.

3. Pincus JH, Cooper JR, Turner VL, et al. (1979) Urine test in SNE. Neurology 29: 424-425.

4. Zangen A, Shainberg A (1997) Thiamine deficiency in cardiac cells in culture. Biochem Pharmacol 54: 575-582.

5. Bailey SW, Ayling JE (2009) The extremely slow and variable activity of dihydrofolate reductase in human liver and its implication for high folic acid intake. Proc Natl Acad Sci U S A 106: 15424-15429.

6. Schenk G, Duggleby RG, Nixon PF (1998) Properties and functions of the thiamin diphosphate dependent enzyme transketolase. Int J Biochem Cell Biol 30: 1297-1318.

7. Deth RC (2013) Autism: a redox/methyhlation disorder. Glob Adv Health Med 2: 68-73.

8. Frye RE, Rossignol DA (2014) Treatments for biomedical abnormalities associated with autistic spectrum disorder. Front Pediatr 2: 66.

9. Giugliani ER, Jorge SM, Goncalves AL (1985) Serum vitamin B12 levels in parturients, in the intervillous space of the placenta and in full-term newborns and their interrelationships with folate levels. Am J Clin Nutr 41: 330-335.

10. Jun WY, Ann YK, Kim JY, et al. (2017) Kawasaki disease with fever and cervical lymphadenopathy as the sole initial presentation. Korean Circ J 47: 107-114.

11. Kohrogi K, Imagawa E, Muto $Y$, et al. (2015) Biotin-responsive basal ganglia disease: A case diagnosed by whole exome sequencing. J Hum Genet 60: 381-385.

12. Malik ZA, Abadi J, Sansary J, et al. (2009) Elevated levels of vitamin B12 and folate in vertically infected children with HIV-1. AIDS 23: 403-407. 
Citation: Lonsdale D (2018) The Dilemma of Raised Blood Levels of Folate and B12 in Autism. J Neurodegener Disord 2(1):35-38

13. Butterworth RF, Gaudreau C, Vincelette J, et al. (1991) Thiamine deficiency and Wernicke's encephalopathy in AIDS. Metab Brain Dis 6: 207-212.

14. Anwar A, Marini M, Abruzzo PM, et al. (2016) Quantitation of plasma thiamine, related metabolites and plasma protein oxidative damage markers in children with autism spectrum disorder and healthy controls. Free Radic Res 50: S85-S90.

15. Aeschbach R, Amado R, Neukom H (1976) Formation of dityrosine cross-links in proteins by oxidation of tyrosine residues. Biochimica et Biophysica Acta (BBA) - Protein Structure 439: 292-301.

16. Obrenovich ME, Shola D, Schroedel K, et al. (2015) The role of trace elements, thiamin (e) and transketolase in autism and autistic spectrum disorder. Front Biosci (Elite Ed) 7: 229-241.

17. Shamberger RJ (2011) Autism rates associated with nutrition and the WIC program. J Am Coll Nutr 30: 348-353.

18. Baird JS, Ravindranath TM (2015) Vitamin B deficiencies in the critically ill autistic child with a restricted diet. Nutr Clin Pract 30: 100-103.
19. Simon N (1990) Infantile autism and Wernicke's encephalopathy. Med Hypotheses 32: 169-172.

20. Malhi P, Venkatesh L, Bharti B, et al. (2017) Feeding problems and nutrient intake in children with and without autism: A comparative study. Indian J Pediatr 824: 283-288.

21. Lonsdale D, Shamberger RJ, Audhya T (2002) Treatment of autistic spectrum children with thiamin tetrahydrofurfujryl disulfide: A pilot study. Neuro Endocrinol Lett 23: 303-308.

22. Hills JI, Golub MS, Bettendorff L, et al. (2012) The effect of thiamin tetrahydrofurfuryl disulfide on behavior of juvenile DBA/2J mice. Neurotoxicol Teratol 34: 242-252.

23. Babaei-Jadidi R, Karachalias N, Ahmed N, et al. (2003) Prevention of incipient diabetic nephropathy by high-dose thiamin and benfotiamine. Diabetes 52: 2110-2120.

24. Rabbani N, Thornalley PJ (2011) Emerging role of thiamine therapy for the prevention and treatment of early-stage diabetic nephropathy. Diabetes Obes Metab 13: 577-583.

25. Page GL, Laight D, Cummings MH (2011) Thiamine deficiency in diabetes mellitus and the impact of thiamine replacement on glucose metabolism and vascular disease. Int J Clin Pract 65: 684-690. 